\title{
Efecto de la temperatura en la respuesta electroquímica en electrolizador tipo PEM utilizando como ánodo Pt-IrO $/$ Ebonex-Ta
}

\author{
J.C. Cruz ${ }^{\mathrm{a} *}$, R. Mena-Rivero ${ }^{\mathrm{a}}$, E. Fuentes Quezada ${ }^{\mathrm{b}}$ \\ ${ }^{a}$ División de Estudios de Posgrado e Investigación, Instituto Tecnológico de Chetumal, Av. Insurgentes No. 330, C.P. \\ 77013, Col. David Gustavo Gtz., Chetumal, Quintana Roo, México. \\ ${ }^{b}$ Centro de Investigación y Desarrollo Tecnológico en Electroquímica S.C., Parque Tecnológico Querétaro, Sanfandila, \\ 76703 Pedro Escobedo, Querétaro, México. \\ *E-mail: jcca12345@hotmail.com
}

Recibido 30 Agosto 2013, Aceptado 25 Septiembre 2013

\section{Resumen}

En el presente trabajo se determinaron los parámetros electrocinéticos y termodinámicos de un electrodo dual en el compartimiento del $\mathrm{O}_{2}$ en modo electrolizador utilizando como catalizadores $\mathrm{Pt}_{-} \mathrm{IrO}_{2}$ soportado en Ebonex dopado con Tantalio para la reacción de evolución de $\mathrm{O}_{2}$ y tela de carbón con $\mathrm{Pt}$ al 30\% soportado en C/Vulcan para el compartimiento de hidrógeno. Las pruebas de polarización y los espectros de impedancia electroquímica se realizaron variando la temperatura de operación de $27-80^{\circ} \mathrm{C}$; se observó una mejora en el desempeño de la celda en la zona de activación y en la zona mixta, se obtuvo una máxima densidad de corriente de $1.15 \mathrm{~A} @ 1.8 \mathrm{~V}$ a una temperatura de $80^{\circ} \mathrm{C}$. Y una menor resistencia en serie a temperatura de $80^{\circ} \mathrm{C}$ siendo esta $0.33 \Omega \mathrm{cm}^{2}$ y una baja resistencia a la transferencia de carga obteniendo $1.74 \Omega \mathrm{cm}^{2}$, de igual manera se verificó su estabilidad mediante cronoamperometría durante 40 minutos.

Palabras clave: Ebonex dopado con Ta, $\mathrm{Pt}-\mathrm{IrO}_{2}, \mathrm{REO}$

\section{Abstract}

In this work, the electrokinetic and thermodynamic parameters of a dual electrode $\mathrm{O}_{2}$ compartment electrolyzer mode using Pt-IrO $\mathrm{I}_{2}$ catalysts supported on Tantalum doped Ebonex for $\mathrm{O}_{2}$ evolution reaction (OER) and carbon cloth with $\mathrm{Pt}$ $30 \%$ supported on C / Vulcan for hydrogen compartment. Polarization tests and electrochemical impedance spectra were performed by varying the operating temperature of 27-80 ${ }^{\circ} \mathrm{C}$, showed an improvement in cell performance in the activation zone and in the mixed zone, the maximum current density was obtained from 1.8V@1.15A at a temperature of $80^{\circ} \mathrm{C}$. And a lower series resistance at $80^{\circ} \mathrm{C}$ being $0.33 \Omega \mathrm{cm}^{2}$ and this low resistance to charge transfer obtaining $1.74 \Omega \mathrm{cm}^{2}$, similarly checked for stability by chronoamperometry for 40 minutes.

Keywords: Ta-Doped Ebonex, Pt-IrO2, OER

\section{Introducción}

Un electrolizador es una celda electrolítica que consiste en un sistema no espontáneo debido a que la reacción que se lleva a cabo en él presenta una variación en la energía libre de Gibbs mayor a cero $(\Delta \mathrm{H}>0)$. Por tal razón, para lograr la electrolisis del agua es necesario suministrar una corriente eléctrica a través de una fuente externa. La corriente eléctrica requerida por el electrolizador para llevar a cabo la reacción química no espontánea, se alimenta a través de dos electrodos (ánodo y cátodo) los cuales se encuentran en contacto con agua y los electrocatalizadores depositados sobre la superficie de la membrana polimérica (electrolito). La electrolisis del agua es un proceso que permite descomponer la molécula en los dos elementos que la constituyen (hidrógeno y oxígeno).

Por el lado del ánodo se lleva a cabo la oxidación del agua, dando como resultado la liberación de oxígeno sobre la superficie del catalizador, la liberación de electrones así como también de iones positivos $(\mathrm{H}+)$. Los electrones circulan a través del circuito externo mientras que los iones $\mathrm{H}+$ se difunden a través del electrolito hacia el cátodo, de acuerdo con la siguiente reacción:

Reacción Global: $2 \mathrm{H}_{2} \mathrm{O}_{(\mathrm{l})} \rightarrow 2 \mathrm{H}_{2}+\mathrm{O}_{2}$
En el cátodo se lleva a cabo la reducción de los protones provenientes del ánodo, que en presencia de los electrones provenientes del circuito externo forman hidrógeno molecular en forma gaseosa que es liberado, como se ilustra en la Fig. 1.

La reacción global que se lleva a cabo en el electrolizador con membrana polimérica es la siguiente:

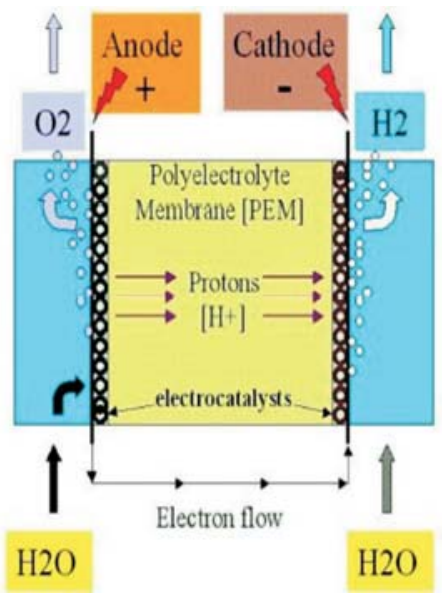

Figura 1. Esquema de un electrolizadador tipo ESP [7].
$\mathrm{E}_{\text {Celda }}^{\circ}=\mathrm{E}_{\mathrm{C}}^{\circ}-\mathrm{E}^{\circ \mathrm{A}}=(0-1.23) \mathrm{V}=-1.23 \mathrm{~V}$ 


\subsection{Termodinámica del electrolizador.}

En condiciones estándar de temperatura y presión $\left(25^{\circ} \mathrm{C}\right.$ y $\left.1 \mathrm{~atm}\right)$ se sabe que:

$$
\Delta \mathbf{G}^{\circ}=-\mathbf{n F E}^{\circ} \text { Celda }
$$

Sustituyendo los valores correspondientes tenemos:

$\Delta \mathrm{G}^{\circ}=-(2)\left(96,484 \mathrm{~J} / \mathrm{V}^{*} \mathrm{~mol}\right)(-1.23 \mathrm{~V})=237,350 \mathrm{~J} / \mathrm{mol}$

La cantidad teórica de energía libre total para llevar a cabo electrólisis del agua está dada por la ecuación 3 y es equivalente a la entalpía de formación $\left(\Delta \mathrm{H}^{\circ}\right)$, que en condiciones estándar tiene un valor de $286 \mathrm{~kJ} / \mathrm{mol}$. Esta energía demandada está relacionada con el voltaje termoneutral de la celda (fig. 2).

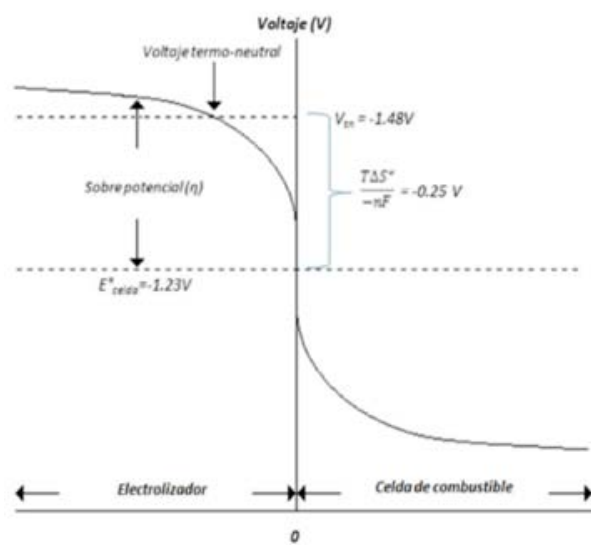

Figura 2. Comportamiento integrado V vs I de un electrolizador y una celda de combustible.

$$
\begin{aligned}
& \Delta \mathbf{H}^{\circ}=\Delta \mathbf{G}^{\circ}+\mathbf{T} \Delta \mathbf{S}^{\circ} \\
& \mathbf{V}_{\mathrm{tn}}^{\circ}=\Delta \mathbf{H}^{\circ} / \mathbf{n} \mathbf{F}
\end{aligned}
$$

Sustituyendo los valores obtenemos :

$$
\mathrm{V}_{\mathrm{tn}}^{\circ}=\frac{286 \mathrm{~kJ} / \mathrm{mol}}{-2 * 96.484 \frac{\mathrm{kJ}}{\mathrm{V}} * \mathrm{~mol}}=-1.48 \mathrm{~V} \text { (4.a) }
$$

Así, para asegurar la electrólisis del agua, es necesario aplicar unsobrepotencial mayor al termo neutral. El Pt parece ser el único catalizador capaz de generar altas velocidades de reacción a temperaturas relativamente bajas $\left(80^{\circ} \mathrm{C}\right)$, la escasa abundancia de platino y los altos costos hacen de esta opción un camino poco viable generando la necesidad de estudiar nuevos materiales. Por tal razón, los investigadores trabajan en encontrar catalizadores más eficientes y baratos que permitan la comercialización de estos dispositivos.

Uno de los materiales utilizados por su bajo sobrepotencial para llevar a cabo la Reacción de Evolución de Oxígeno (REO) es el $\mathrm{IrO}_{2}$, en un rango de 0.27-0.50 V, de acuerdo al diagrama de Volcano reportado por Trasatti [1]. Este material es utilizado para ánodos dimensionalmente estables (DSA $®)$ en el cual a tenido mayor impacto [2-6].

Debido a que el $\mathrm{IrO}_{2}$ contiene la propiedad, como catalizador, de una alta resistencia a la corrosión en un medio ácido a diferencia de el $\mathrm{RuO}_{2}$, sin embargo la actividad electrocatalítica del $\mathrm{IrO}_{2}$ para llevar la $\mathrm{REO}$ no es superior, se han reportado un gran avance en cuanto al uso de este material en un medio ácido para llevar a cabo dicha reacción [7-9].

Recientemente el $\mathrm{IrO}_{2}$ está siendo examinado para su uso en electrolizadores del tipo ESP [10], obteniéndolos mediante algunos métodos de síntesis como son: el de fusión de Adam [11], sol-gel [12-15], poliol [6,16]. Los diferentes métodos de obtención de este óxido pueden afectar las propiedades catalíticas del material. Por esta razón se han estudiado y comparado estas diferentes metodologías de obtención de estos materiales, partiendo de distintas sales.

En este trabajo se pretende usar un material Pt-IrO $\mathrm{I}_{2}$ soportado en Ebonex dopado con Tantalio, con el objeto de reducir las cargas catalíticas de los materiales preciosos soportándolo en un material menos costoso con el objeto de minimizar costos e incrementar el área electroactiva. Se obtuvieron los parámetros cinéticos y electroquímicos en una celda de $5 \mathrm{~cm}^{2}$ con respecto a la variación de temperatura de entrada del fluido (agua desionizada) en un rango de $27-80^{\circ} \mathrm{C}$.

\section{Parte experimental}

\subsection{Preparación de ensamble membrana electrodo}

La tinta catalítica que se utilizó para la construcción de los ensambles estuvo constituida por una mezcla 50:50 de material catalítico y material soporte, el primero corresponde al $\mathrm{Pt}-\mathrm{IrO}_{2}$ (con una composición, a su vez, 50:50) y el segundo de $\mathrm{TinO}_{2 \mathrm{n}-1}-\mathrm{Ta}$ (Ebonex dopado con Ta), respectivamente. Se utilizó además tela difusora de gas (GDL's) suministrada por E-Tek, así como, una solución de Nafión al 5\% marca Aldrich ${ }^{\circledR}$. La membrana empleada en los ensambles fue Nafión 115 adquirida por Ion Power ${ }^{\circledR}$.

La membrana se pre-trato en cuatro diferentes baños calientes. El primer baño se realizó en una solución de $\mathrm{H}_{2} \mathrm{O}_{2}$ al $3 \%$, seguido de un enjuague con agua desionizada, cada baño con una duración de una hora.

El tercer y cuarto baño se realizó en una solución $1 \mathrm{M}$ de $\mathrm{H}_{2} \mathrm{SO}_{4}$ seguido de un enjuague en agua desionizada cada uno con una duración de una hora. La temperatura de los baños fue de $80^{\circ} \mathrm{C}$.

Después del pre-tratamiento, las membranas se mantuvieron sumergidas en agua desionizada para su posterior uso.

Para la realización del electrodo anódico se asumió una composición del $67 \%$ de material catalítico/soporte y $33 \%$ de Nafión, dicha mezcla se diluyó en etanol con la finalidad de facilitar la dispersión de las partículas. La cantidad de soporte (Ebonex dopado con Ta), así como de material catalítico que se peso fue de $10.9 \mathrm{mg}$. De acuerdo con la composición del $\mathrm{Pt}-\mathrm{IrO}_{2}, 5.45 \mathrm{mg}$ corresponden al Pt y $5.45 \mathrm{mg}$ al $\mathrm{IrO}_{2}$. La cantidad de Nafión al 5\% que se utilizó para la tinta fue de 214.74 mg $(250 \mu \mathrm{L}$ que corresponden al $33 \%$ de la composición 
de la tinta). La tinta resultante se colocó en un baño ultrasónico durante 1 hora, para después realizar el depósito de la misma sobre la membrana, utilizando la técnica de rociado en caliente con aerógrafo.

Para conocer la carga catalítica, esta se determinó por pesado. El peso de la membrana sin depósito resulto de $1.7262 \mathrm{~g}$ y el peso con depósito fue de $1.7298 \mathrm{~g}$. Por diferencia de estas dos cantidades, se determinó la cantidad catalítica depositada, la cual resulto de $3.6 \mathrm{mg}$. De esa cantidad, solo $2.41 \mathrm{mg}(67 \%)$ corresponde al material catalítico soportado. Ya que el área del ensamble en que se depositó la tinta fue de $5 \mathrm{~cm} 2$, la carga catalítica corresponde a $05 \mathrm{mg} / \mathrm{cm}^{2}$.

Para el lado anódico del ensamble, se utilizó tela difusora de gas (tela de carbón) con una carga catalítica de $0.77 \mathrm{mg} / \mathrm{cm}^{2}$ de $\mathrm{Pt} / \mathrm{C} 30 \%$. Construido el ensamble, esté se prenso en caliente a una temperatura de $130^{\circ} \mathrm{C}$ y una presión de 75 psi durante 90 segundos utilizando una prensa hidráulica Autofour con placas de calentamiento programables marca Carver. Sinterizado el ensamble, este se colocó en la celda de pruebas utilizando dos empaques, la presión de torque a la que se sometió la celda fue de 25 psi.

\subsection{Caracterización electroquímica}

Se utilizó una estación de pruebas ElectroChem, Inc. (Power Station, System Interface) acoplado a un controlador de flujos de la misma marca. Para los estudios de espectroscopia de impedancia, se utilizó un Potenciostato-Galvanostáto AutoLab acoplado a un Booster 20 A ambos controlados por un software de computadora.

El flujo de agua desionizada fue de $5 \mathrm{ml} \mathrm{min}^{-1}$ a presión ambiente. Las temperaturas a las que se sometió la celda fueron de $27,40,60$ y $80{ }^{\circ} \mathrm{C}$ y de igual manera la entrada de la temperatura del fluido fue controlada mediante la estación de prueba con el objeto de disminuir una variación de la misma.

\section{Resultados y Discusión}

El primer barrido de potencial a temperatura ambiente (fig. 3) se puede observar la existencia de dos picos, los cuales corresponden, el primero a la formación de Pt-O (el Pt adsorbe moléculas de oxígeno) y el segundo a la formación de óxidos de $\mathrm{Pt}\left(\mathrm{Pt}-\mathrm{O}_{2}\right)$ que posteriormente, al incrementar el potencial el Pt libera para formar $\mathrm{O}_{2}$ molecular.

Para el segundo y tercer barrido, los picos van disminuyendo hasta ya no notarse, esto se debe a que el Pt ha saturado todos sus sitios activos con la adsorción de oxígeno. También se nota, que al tercer barrido, la corriente que se registra es mayor que los dos barridos anteriores, esto se relaciona directamente con la transferencia de masa, ya que al formarse los óxidos, se produce mucha más cantidad de oxígeno molecular.

Después de saturar los sitios activos del Pt, se realizaron curvas de polarización incrementando la temperatura a 40,60 y $80{ }^{\circ} \mathrm{C}$ (fig. 4) con un barrido anódico de potencial desde 0.6 hasta $1.8 \mathrm{~V}$ observando que a potenciales mayores al termoneutral $(1.48 \mathrm{~V})$ comienza la evolución del medio, siendo más evidente a temperaturas de 40,60 y $80^{\circ} \mathrm{C}$. Se obtuvo una respuesta de $1.15 \mathrm{~A} @ 1.8 \mathrm{~V}$ a una $\mathrm{T}$ de $80^{\circ} \mathrm{C}$. El incremento de corriente, está asociado a la producción de hidrógeno (transferencia de masa), en donde es importante mencionar que a $27{ }^{\circ} \mathrm{C}$, esta corriente es muy pequeña respecto a las demás, es decir, poca producción de gas.

Conforme aumenta la temperatura podemos notar que el potencial que se requiere para la evolución del medio es menor y la corriente se incrementa.

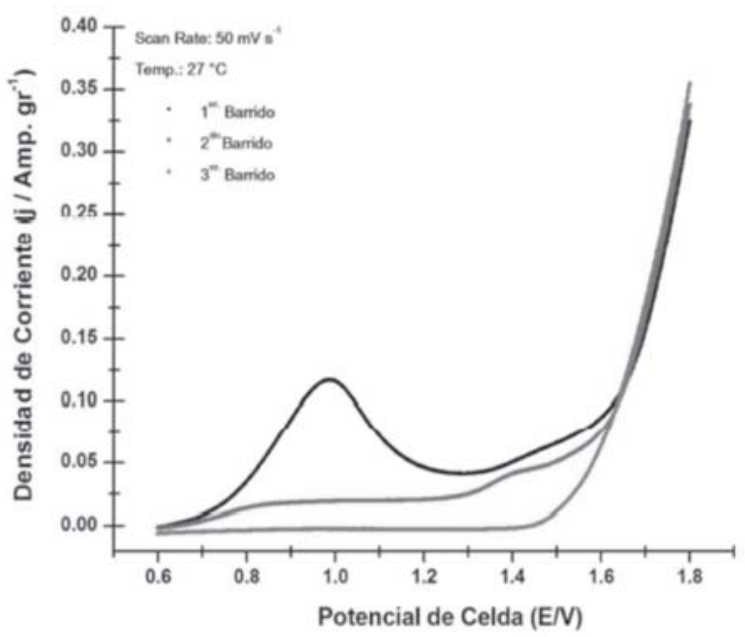

Figura 3. Curvas de Polarización en Modo Electrolizador a diferentes barridos y a una temperatura de operación de $27^{\circ} \mathrm{C}$.

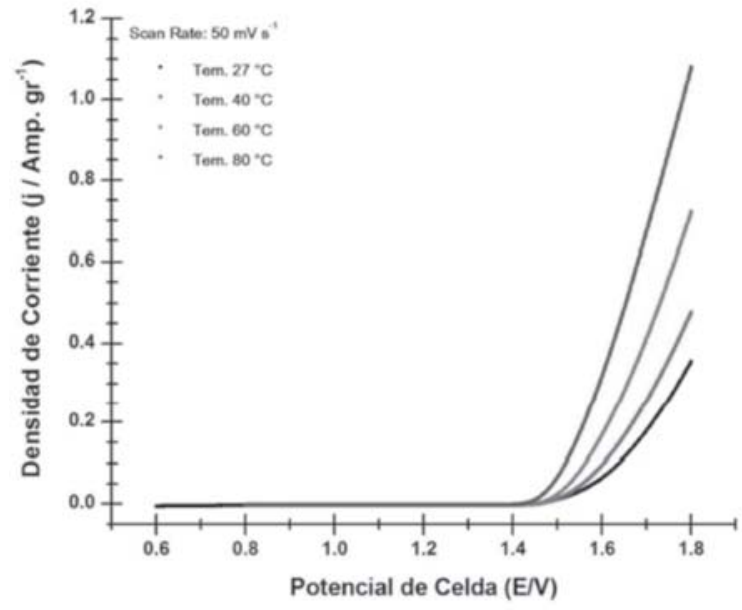

Figura 4. Curvas de Polarización en Modo Electrolizador a diferentes temperaturas de operación

Se realizó un estudio de impedancia electroquímica a la respuesta de la variación de la temperatura, en la fig. 5 se puede observar los diagramas de Nyquist obtenido en modo electrolizador puede observarse que a medida que la temperatura aumenta, la resistencia a la transferencia de carga disminuye. Para las temperaturas de 60 y $80^{\circ} \mathrm{C}$, los puntos se contraponen pudiéndose atribuir al acumulo de burbujas en la superficie del electrodo, es por ello que solo se observa los datos de la temperatura de $60{ }^{\circ} \mathrm{C}$. 


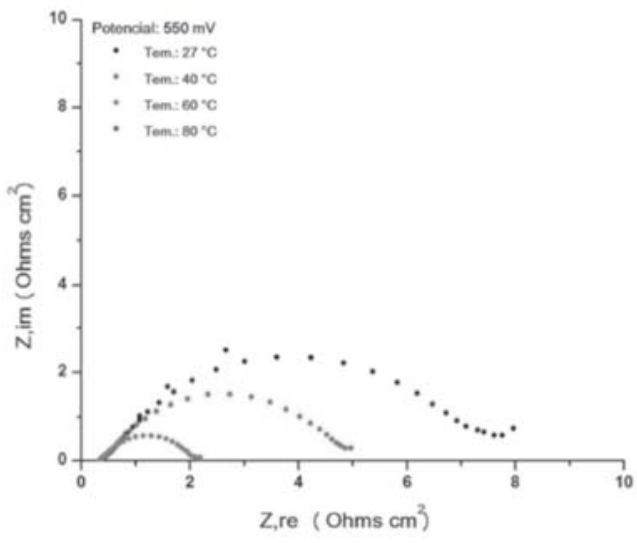

Figura 5. Diagrama de Nyquist para MEA de $5 \mathrm{~cm}^{2}$ de área superficial, construido con Pt-IrO2/Ebonex-Ta y Pt/C 30\% diferentes temperaturas de operación.

En la tabla 1 se muestran el resumen de las resistencias en serie y la transferencia de carga a diferentes temperaturas para el modo electrolizador. Cabe mencionar, que los valores de dicha tabla se obtuvieron prolongando los semicírculos hasta que estos se interceptarán con el eje de las coordenadas, es decir, de manera gráfica.

Tabla 1. Resistencias en serie y de transferencia de carga a diferentes temperaturas de operación en modo electrolizador.

\begin{tabular}{lll}
\hline Temp. $\left({ }^{\circ} \mathrm{C}\right)$ & $\mathrm{R}_{\text {serie }}\left(\Omega \mathrm{cm}^{2}\right)$ & RTC $(\Omega \mathrm{cm} 2)$ \\
\hline 27 & 0.39 & 7.72 \\
40 & 0.37 & 4.26 \\
60 & 0.33 & 1.74 \\
80 & 0.33 & 1.74 \\
\hline
\end{tabular}

Para determinar la estabilidad del electro-catalizador, se realizó una cronoamperometría a un potencial de 1.55 $\mathrm{V}$, la cual se muestra en la fig. 6. Se pude observar que a ese potencial y durante ese tiempo es estable; sin embargo, se recomienda realizar un estudio más profundo sobre la estabilidad del material mediante técnicas de multiciclos.

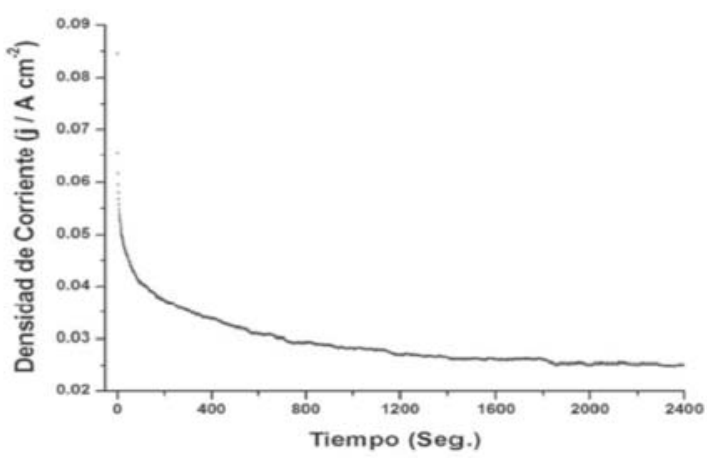

Figura 6. Cronoamperograma a un potencial fijo de $1.55 \mathrm{~V}$ durante 40 minutos operando en modo Electrolizador
Ya que para el modo electrolizador, las reacciones que se llevan a cabo son inversas a las producidas en modo celda de combustible, es decir, se parte de agua para producir hidrógeno y oxígeno, los parámetros termodinámicos serán de la misma magnitud pero con signo contrario, así entonces, tenemos las respuesta a las variaciones de energía libre de Gibbs, entalpia, entropía y potencial de activación (tabla 2), cabe mencionar que esta última se representa con signo negativo, sin embargo, es debido a la necesidad de aplicación de este potencial a manera anódica siendo un ejemplo la necesidad de aplicación de $1.466 \mathrm{~V}$ a $80^{\circ} \mathrm{C}$ para el inicio de la REO.

Tabla 2. Parámetros teóricos termodinámicos obtenidos para diferentes temperaturas de operación en modo Electrolizador.

\begin{tabular}{ccccc}
\hline $\begin{array}{c}\text { Temp. } \\
(\mathrm{K})\end{array}$ & $\begin{array}{c}\Delta \mathrm{H} \\
\mathrm{kJ} \mathrm{mol}^{-1}\end{array}$ & $\begin{array}{c}\Delta \mathrm{S} \\
\mathrm{kJ} \mathrm{mol}^{-1} \mathrm{~K}^{-1}\end{array}$ & $\begin{array}{c}\Delta \mathrm{G} \\
\mathrm{kJ} \mathrm{mol}^{-1}\end{array}$ & $\begin{array}{c}\mathrm{E}_{\mathrm{Tn} .} \\
\text { Volts }\end{array}$ \\
\hline 298.15 & 285.07 & 0.1636 & 236.29 & -1.477 \\
\hline 300.15 & 285.00 & 0.1633 & 235.98 & -1.476 \\
\hline 313.15 & 284.50 & 0.1617 & 233.87 & -1.474 \\
\hline 333.15 & 283.74 & 0.1593 & 230.67 & -1.470 \\
\hline 353.15 & 282.96 & 0.1571 & 227.48 & -1.466 \\
\hline
\end{tabular}

\section{Conclusiones}

Se realizó una caracterización electroquímica en modo de celda de electrolizador utilizando $\mathrm{Pt}-\mathrm{IrO}_{2} / \mathrm{Ebonex}^{-\mathrm{Ta}}$ en el compartimiento del electrodo de oxígeno y se obtuvieron los parámetros electrocinéticos y termodinámicos en un régimen de temperatura de 27$80{ }^{\circ} \mathrm{C}$. Se pudo observar que la temperatura afecta a la zona de activación al incremento de esta, disminuyendo la energía necesaria favoreciendo la zona de activación y la zona mixta, inversamente a una celda de combustible, Se obtuvo una respuesta de 1.15 A @ 1.8V a una T de $80^{\circ} \mathrm{C}$. Y una menor resistencia en serie a temperatura de $80^{\circ} \mathrm{C}$ siendo esta $0.33 \Omega \mathrm{cm}^{2}$ y una baja resistencia a la transferencia de carga siendo esta $1.74 \Omega \mathrm{cm}^{2}$.

\section{Referencias}

1. L.M. Da Silva, J.F.C. Boodts, Electrochim. Acta, pg. 1369-1375 (2001).

2. H. Wendt, Electrochemical Hydrogen Technologies, Elsevier, Holanda (1990).

3. HB Beer, US patent US549194, (1966); US710551,(1968).

4. S. Ardizzone, A. Carugati, S. Trasatti , Journal Electroanal. Chem. 126:287 (1981).

5. J. Kristof, Mink J., Electrochim. Acta 39,1531(1994)

6. A. Benedetti, P. Riello, G. Battaglin, A. De Battisti, A. Barbieri, J. Electroanal. Chem 376:195 (1994).

7. AJ. Terezo, EC .Pereira, Electrochim Acta, 45, 4351 (2000).

8. GP. Vercesi, JY. Salamin, Ch. Cominellis, J. Appl Electrochem., 21, 335, (1991) 
9. MI. Rojas, MJ. Eslandiu, LB. Avalle, EPM. Leiva, VA. Macagno, Electrochim Acta,43, 1785,(1998).

10. JM. Hu, JQ. Zhang, HM Meng, CN Cao, J. Mater Sci., 38,705,( 2003).

11. L. Morales S., L. G. Arriaga, C. Ucano., R. Acosta., ECS Transactions, 3, (2006).

12. R. Adams, R. Shriner, J. Am. Chem. Soc. , 45, 2171 (1923).

13. Y. Murakami, S. Tuschiya, K. Yaahikozawa, Y. Takasu, Electrochim. Acta, 39, 651 (1994).

14. M. Ito, Y. Murakami, H. Kaji, H. Ohawauchi, K. Yahikozawa, Y. Takasu, J. Electrochem. Soc., 141, 1243 (1994).

15. K. Kameyama, S. Shohji, S. Onoue, K. Nishimura, K. Yahikozawa, Y. Takasu, J. Electrochem. Soc., 140, 1034 (1993).

16. T. Lassali, J. Boodts, L. Bulhoes, J. Non-Crist. Solids, 273,129 (2000). 